\title{
INFLUENCE OF THE PREPARATION DESIGN AND AGING ON THE VERTICAL MARGINAL GAP OF OCCLUSAL VENEERS CONSTRUCTED OF DIFFERENT CERAMIC MATERIALS
}

\author{
Manal Rafei Hassan Abo-Eittah ${ }^{*}$ and Mohammed Moustafa Shalaby**
}

\begin{abstract}
Aim: The current study aimed to investigate the marginal adaptation of two different designs of occlusal veneers manufactured from two different ceramic materials before and after aging.

Material and Methods: Twenty freshly extracted mandibular second molars were used in this study. The teeth were randomly divided into 2 groups according to the occlusal veneer material $(\mathrm{n}=10$ each). The first group in which the teeth were restored with occlusal veneers constructed of reinforced lithium silicate (ZLS), (Celtra Duo, Dentsply Sirona, Germany), while the second group, the occlusal veneer material was the polymer-infiltrated hybrid ceramic (Vita Enamic, Vita Zahnfabrik, Germany). Each group was further subdivided into 2 subgroups ( $\mathrm{n}=5$ each) according to the preparation design. In the first subgroup, the reduction of teeth involved conventional occlusal reduction (planner preparation). In the second subgroup, the reduction of teeth involved the occlusal surface plus $1 \mathrm{~mm}$ axial reduction with rounded shoulder finish line. The occlusal veneers were designed and milled using $\mathrm{CAD} / \mathrm{CAD}$ technology. The vertical marginal gap was measured using stereomicroscope before and after the cemented teeth-veneer assemblies were imbedded in food/oral simulating liquids, and thermocycled. Two samples of the constructed occlusal veneers of the two studied materials were examined before and after ageing by FT-IR spectroscopy technique. Results of marginal gap were collected, tabulated and statistically analyzed using independent t-test, and 3way Anova for the interaction between different variables results were considered significant at $\mathrm{p} \leq 0.05$.
\end{abstract}

Results: the results showed that the marginal gap of the two preparation designs of the two materials were statistically non significant before ageing $\mathrm{P} \leq 0.05$. while after ageing, the subgroups of Celtra recorded, $90.45 \pm 13.0$ and $88.7 \pm 11.60 \mu \mathrm{m}$, for conventional and, rounded shoulder designs respectively, with no significant difference between the two designs, while the subgroups of Vita Enamic recorded $113 \pm 7.01 \mu \mathrm{m}$ and $105.2 \pm 8.01 \mu \mathrm{m}$ for conventional and, rounded shoulder designs respectively, with high significant difference $\mathrm{p} \leq 0.003^{* *}$. The Anova test revealed that the interaction between the materials, preparation designs and the ageing procedures, recorded very significant influence on the marginal gap discrepancy.

Conclusions: The choice of certain preparation design should be associated with meticulous knowledge of the material of the occlusal veneers properties .Both materials could be used in occlusal veneers restorations.Celtra Duo had a marginal adaptation that survived with better values, qualitatively and quantitatively, even after the protocol of ageing applied in this study.

* Associate professor and Head of Fixed Prosthodontics Department, Faculty of Dentistry, Minia University, Egypt.

** Lecturer of Fixed Prosthodontics, Fixed Prosthodontics Department, Faculty of Dentistry, Minia University, Egypt. 


\section{INTRODUCTION}

Tooth wear may be attributed to several factor such as the dietary habits, medical status, and/or oral habits. These factors can cause attrition, abrasion, and erosion of the tooth structure ${ }^{(1)}$. This tooth damage is of great worry as it affects the musculoskeletal harmony, occlusal harmony, esthetics, and overall the patients contentment towards their own teeth ${ }^{(2,3)}$.

The treatment options for restoring severely worn down teeth may involve crowns, surgical crown lengthening, and optional tooth devitalization ${ }^{(4)}$. The main driving force in restorative dentistry is the preservation of as much as possible sound tooth structure ${ }^{(5,6)}$. From a biomimetic perspective, the conservation of tooth structure is vital in maintaining the subtle balance between biologic, mechanical, and esthetic factors ${ }^{(7)}$. Keeping the pulp vital, avoiding the root canal treatment and the need for post and cores is of great concern. As these more invasive approaches compromise the function and esthetics of the restored teeth over time ${ }^{(7,8)}$.

It have been reported that, partial coverage preparations such as onlay, partial coverage ceramic crowns require the removal of $40 \%$ of the tooth structure if compared to full coverage crowns ${ }^{(6)}$. One of the most current conservative treatment options is the occlusal veneer. Posterior occlusal veneers are extra-coronal restorations that requires lesser preparation, this is governed by inter occlusal space and the variations in the anatomy. Occlusal veneers are indicated in case of reduction in the thickness of the occlusal enamel, so the underlying dentin is exposed at the occlusal surface.

It was found that the combination of CAD/CAM technology and a state of the art bonding protocol resulted in paradigm shift regarding the treatment of wear cases where the space is limited ${ }^{(9,10)}$.

Occlusal veneers can be manufactured from different ceramic materials. Among the available glass ceramic materials, it was found that Lithium disilicate ceramics has the highest mechanical properties. This may be contributed to their microstructure that involves interlocking, needlelike crystals embedded in the glassy matrix ${ }^{(11)}$. Ten percent zirconia is added to the glass matrix to enhance the mechanical properties ${ }^{(12)}$.

In another line for better mechanical properties. a ceramic network structure was infiltrated with a polymer material to gain the benefits of the two materials. The mechanical and physical properties of the machinable polymer restorative materials have been enhanced that allowed them to replace glass-ceramics, especially when thin restorations are subjected to heavy occlusal forces, because of their high fatigue resistance ${ }^{(13)}$.

The longevity of these dental materials should be investigated when they are chosen for construction of occlusal veneers. The adaptation of the restoration margin is one of the most vital factors for the survival of the restorations. In order to prevent the dissolution of the cement, and to have a healthy periodontium, a perfect marginal fit must be considered. With the recent advances in CAD/CAM systems, the recorded marginal gap of the prosthesis is a very low value within 100 microns, while most of the literatures agreed that the clinically allowable marginal gap to be within a range of 100-120 microns.

The food/oral simulating liquids have been recommended by the Bureau of Food, FDA. Some of them do exist in the mouth while, others can simulate its conditions (ingredients, $\mathrm{pH}$, viscosity). Their use permits us to see a fast wear of the materials in short time and furthermore to take in consideration processes like chemical affinity, elution or bonding. It goes without saying that physical/mechanical properties are affected profoundly by the presence of the food simulating liquids ${ }^{(14)}$. Food simulating fluids can mimic the oral environment; distilled water simulates the wet oral environment provided by saliva and water, while heptane simulates butter, fatty meats and vegetable oils. The citric acid and 
ethanol mimic certain beverages including alcohol, fruits candies and syrups ${ }^{(15)}$.

The aim of this study is to investigate the marginal adaptation of two different designs of occlusal veneers manufactured from two different ceramic materials before and after aging.

The null hypothesis of this study is 1- The marginal gap measurements will not affected either by the preparation design or the ceramic material. 2- The aging will not affect the initial marginal gap measures.

\section{MATERIALS AND METHODS}

Twenty freshly extracted mandibular second molars were collected from the outpatient clinic, Faculty of Dentistry, Minia University. The teeth were used after obtaining the patients' informed consent to their use for research purposes. The teeth were free of caries and fillings and extracted for periodontal purposes. For standardization, the teeth to be included in the present experimentation were measured with a digital caliper and discarded if exceeding the following ranges: crown mesiodistal width, $9.8 \pm 1.0 \mathrm{~mm}$; crown bucco-lingual width, 8.8 $\pm 1.0 \mathrm{~mm}$. The teeth were cleaned from calculous deposits and soft tissues using electric scaler, and stored in a $0.1 \%$ thymol solution. The teeth were embedded in $15 \mathrm{~mm}$ plastic cylinders containing partially- set auto polymerized resin so that the cemento-enamel junction was located $2 \mathrm{~mm}$ above the resin. The teeth were randomly divided into 2 groups according to the occlusal veneer material ( $\mathrm{n}$ $=10$ each). The first group in which the teeth were restored with occlusal veneers made of reinforced lithium silicate (ZLS), (Celtra Duo, Dentsply Sirona, Germany), while in the second group the occlusal veneer material was constructed of the polymer-infiltrated hybrid ceramic (Vita Enamic, Vita Zahnfabrik, Germany). Each group was further subdivided into 2 subgroups ( $\mathrm{n}=5$ each) according to the preparation design. In the first subgroup, the reduction of teeth involved conventional occlusal reduction (planner preparation). In the second subgroup, the reduction of teeth involved the occlusal surface plus $1 \mathrm{~mm}$ axial reduction with rounded shoulder finish line.

\section{Tooth preparation}

All the preparations were done by the same operator. For both subgroups, the occlusal reduction was carried out following the manufacturers' recommendations about the least occlusal thickness for the final restoration. This was applied to the Celtra Duo and the Vita Enamic machinable blocks utilized in this study. Three addition silicone indexes were taken to each tooth before reduction. Figure 1

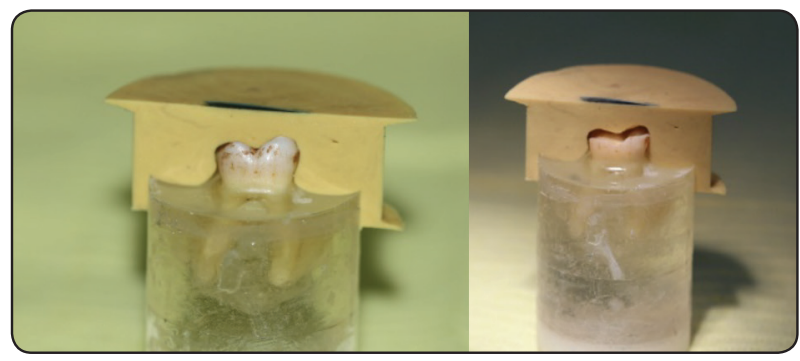

Fig. (1) Silicon indices to standardize the preparation.

The first and second ones were sectioned in a bucco-lingual and mesio-distal directions to check tooth structure removal during preparation. The third one was used as a template for wax pattern fabrication to rebuild the tooth again to verify the amount of reduction in all the surfaces that is equal to the thickness of the wax pattern.

The first subgroup: conventional occlusal reduction (planner preparation). Occlusal preparation: $1 \mathrm{~mm}$ uniform reduction was carried out on the occlusal surface following the anatomy. This uniform reduction was done using short tapered round stone (855D 314 016, Komet, Germany). The stone has a black laser mark at $1 \mathrm{~mm}$ from the instrument tip. The stone was applied to the occlusal surface in a vertical position, and a guide grooves was prepared with depth of $1 \mathrm{~mm}$. Then the tooth structure between the groves was reduced using OccluShaper stones with medium grit (370 Komet). 
The occlusal preparation was completed using finishers of matching shape (8370, Komet).

The second subgroup: The occlusal reduction was carried out as described in the first subgroup. A wider round-ended cylindrical diamond stone (836 KR 314 018, Komet) was utilized to reduce the axial walls creating a rounded shoulder finish line, which was then finished using a fine-grit bur (88836 KR 314 018, Komet).

\section{Restorations design and manufacturing}

Each preparation was then scanned using an intraoral scanner (CEREC Omnicam, Dentsply Sirona, Germany). Then using the in-lab software (CEREC SW 4.4.4., Sirona Dental Systems GmbH, Bensheim, Germany). Each occlusal veneer was designed to rebuild its corresponding tooth. An internal relief spacer of 60 microns was used in all designs for standardization. The design data in the form of STL file was sent to the milling machine (inLab MCXL, Dentsply Sirona, Bensheim, Germany). The occlusal veneers were milled from the desired milling blocks. After milling, the veneers were cut from the sprues, and the connection sites were smoothed. For the Celtra Duo occlusal veneers, they were subjected to crystallization and glazing firing cycles (Programat P310, Ivoclar Vivadent, AG, Schaan, Liechtenstein). Each veneer was then checked for seating on its corresponding tooth. The Vita Enamic occlusal veneers were finished and polished (VITA Enamic Polishing set technical) according to the manufacturer's recommendations.

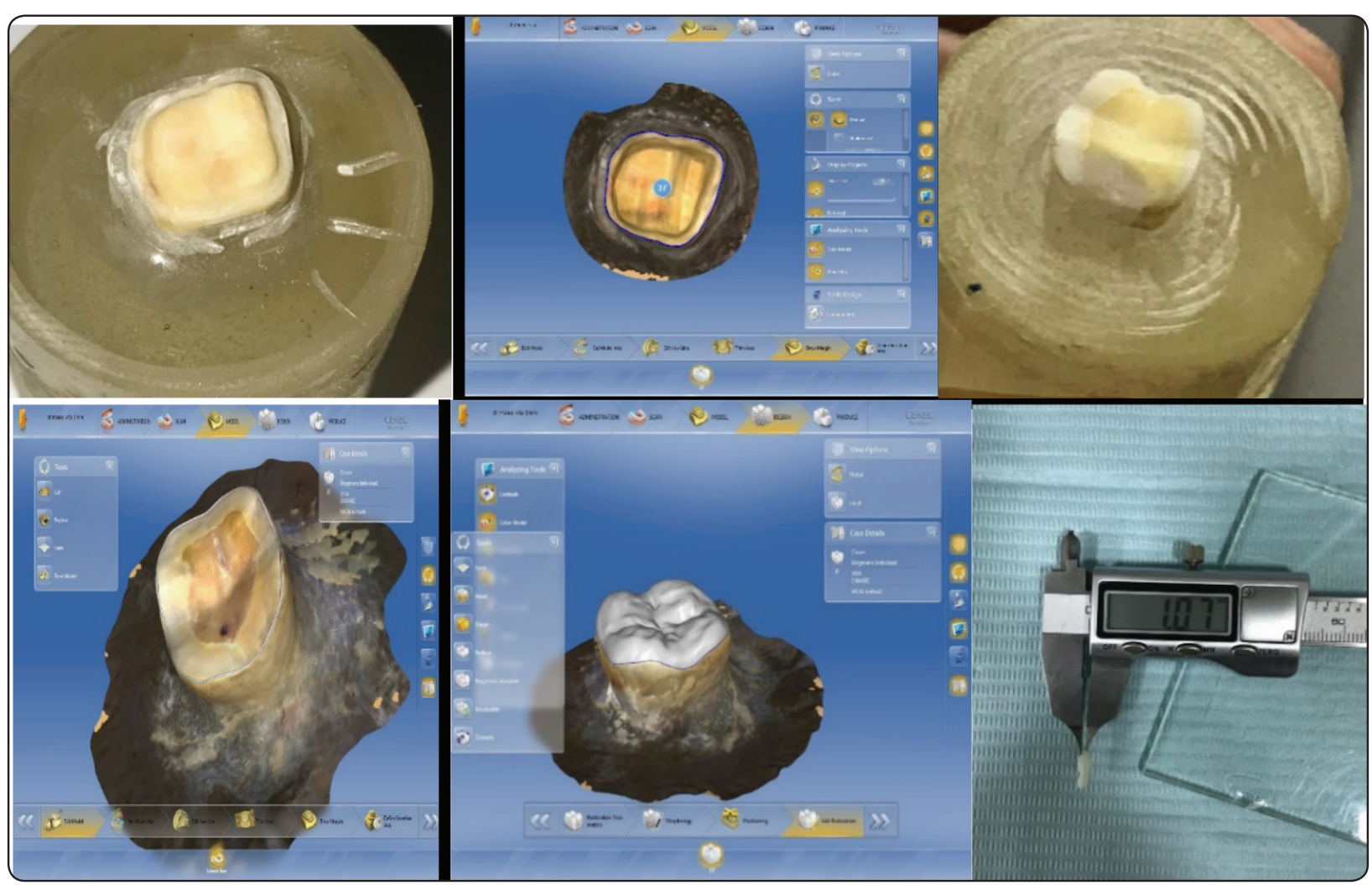

Fig. (2) The two designs of prepared occlusal veneers, and the digital caliber measuring the thickness of the final occlusal veneer $(1.07 \mathrm{~mm})$ 


\section{Cementation of the occlusal veneers}

All the occlusal veneers were cleaned using 99\% isopropanol in an ultrasonic cleaner for 5 minutes. The prepared teeth were cleaned from the preparation debris with fluoride-free pumice (Proxyt RDA 36, medium, Ivoclar Vivadent, AG, Schaan, Liechtenstein) for 15 seconds. After that, they were rinsed thoroughly with water for 15 seconds.

The fitting surface of the occlusal veneers of both materials were etched using $8 \%$ hydrofluoric acid (DENTOBOND Porcelain etch, 4167-29 PFXE, ITENA, Paris, FRANCE) for 30 seconds in the Celtra Duo group and for 60 seconds for the Vita Enamic group. Then the etched sample was properly rinsed with thoroughly water spray and dried using oil-free compressed air. One coat of silane coupling agent (Silane, 4167-29 PFXE, ITENA, Paris, FRANCE) was brushed on the inner surface of the veneers and let to dry for 1 minute. A steam of dry air is applied to remove the remnants of the primer.

The prepared teeth were etched with $37 \%$ phosphoric acid (Total Etch, Ivoclar Vivadent) for 30 seconds, then thoroughly rinsed with water spray for 20 seconds, and dried using oil-free air. Immediately, a tooth primer (Multilink Primer A/B, Ivoclar Vivadent) is mixed for 10 seconds and applied to all the preparation surfaces, thinned with gentle steam of dry air, leaving the surface appearing glossy. Dual-polymerizing composite resin cement (Rely X Ultimate, 3M Espe, Germany) was injected into the fitting surface of the veneers and each veneer was seated on its corresponding preparation with finger pressure. Seating pressure of 49 (equivalent to $5 \mathrm{~kg}$ force) was applied to the veneers by a universal testing machine for 5 minutes. The excess cement was removed using a cotton pellet and the air inhibited gel was applied to the margins. Finally, light curing was applied for each surface for 20 seconds.

\section{Immersion in food simulating liquids}

All samples were immersed, separately, into a tightly closed glass container for 15 days, at room temperature. The components of the Food simulating liquids were; distilled water, $75 \%$ aqueous solution of ethanol, heptane and $0.02 \mathrm{~N}$ citric acid ${ }^{(15)}$.

\section{Thermocycling}

The samples were thermo-cycled for 5000 cycles, each cycle includes immersion for 30 seconds into the hot path at $55 \pm 1^{\circ} \mathrm{C}$ followed by immersion for same time into the cold path at $5 \pm 1{ }^{\circ} \mathrm{C}$ with 5 seconds delay between the hot and cold paths. The vertical marginal gap was measured again for each veneer at the same predetermined points.

\section{Marginal gap evaluation}

Each specimen was photographed using a stereo microscope (Lecia,205MC, USA) connected with an computer monitor screen using a magnification of 7.5 up to $160 \mathrm{X}$. A digital image analysis system (Image J 1.43U, National Institute of Health, USA) was used to measure and evaluate the gap width. Shots of the margins were taken for each specimen, the scale bar was $2 \mathrm{~mm}$. Then morphometric measurements were done for each shot .Ten equidistant landmarks points along the cervical circumference for each surface of the specimen (Mesial, labial, distal, and palatal, a total of 40 points for each sample). Measurement at each point was repeated five times Figure 3. Then the data obtained were collected, tabulated and then, statistically analyzed.

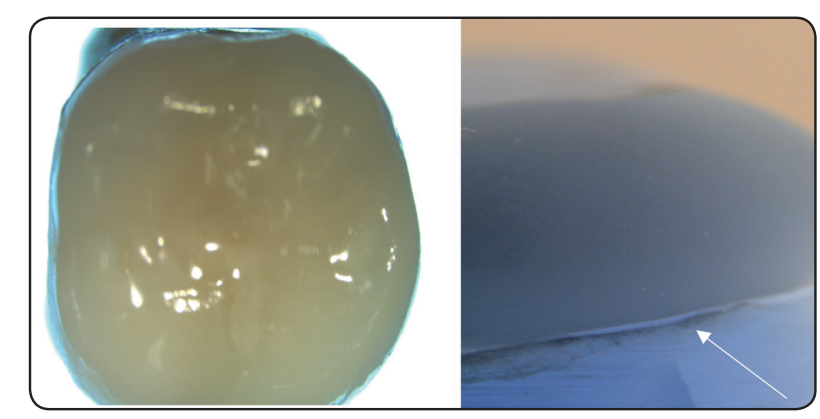

Fig. (3) Occlusal, and axial view of the cemented occlusal veneer. All surfaces will be examined for marginal, quality and vertical gap distance in $\mu \mathrm{m}$. 


\section{Fourier Transform Infrared Spectroscopy (FT-IR) measurements}

Two samples of the constructed occlusal veneers of the two studied materials were examined before and after ageing by FT-IR spectroscopy technique.

Fourier transform infrared spectroscopy was measured to figure out the kind of bonds that may be shifted, disappeared, or degraded inside the ceramics materials after ageing. FTIR instrument identifies chemical bonds in a molecule by production an infrared absorption spectrum. The spectra produce a profile of the sample, a distinctive molecular fingerprint that can be used to screen and scan samples for many different components. Two representative samples from each subgroups were studied with FT-IR. If the spectra of control samples differ than after ageing samples, in term of new bands or shift in existing bands, it denotes new formation of chemical bonds or breakdown of existing ones (new compound with new properties). ATR-FTIR is recorded on JASCO instrument. Samples were scanned at resolution of $4 \mathrm{~cm}-1$ over a wavenumber range of 400-4000 cm-1. Enzyme activity measurements were conducted using UVVis spectrophotometer (JASCO V630, Japan).

FT-IR spectrometer consists of a source, interferometer, sample compartment, detector, amplifier, analog-to-digital converter and a computer. The source generates radiation which passes the sample through the interferometer and reaches the detector. Then the signal is amplified and converted to digital signal by the amplifier and analog-to-digital converter, respectively. Eventually, the signal is transferred to a computer in which Fourier transform is carried out.

FT-IR measurement is the most accurate method that can be used to identify the different functional groups $(\mathrm{O}-\mathrm{H}, \mathrm{N}-\mathrm{H}, \mathrm{C}=\mathrm{C}, \mathrm{C}-\mathrm{H}, \mathrm{Si}-\mathrm{O})$ in the molecule.

\section{RESULTS}

Data were collected tabulated according to the randomization list, then analyzed using IBM SPSS v. 25 to investigate and detect if there were statistical significance differences of the obtained results. Quantitative data were described using range (minimum and maximum), mean, standard deviation and median. Comparison between the mean values of results, by using paired student test within each group to compare between before and after aging results, comparison between groups will be done using independent t-test. Descriptive and inferential statistics will be based upon if collected data considered normally distributed otherwise using nonparametric tests.

Three -way ANOVA used to show the effect of different groups and design on marginal gap, $\mu \mathrm{m}$, of different groups and subgroups followed by multiple comparison with Bonferroni correction Significant level was set at $\mathrm{p} \leq 0.05(\alpha \leq 0.05)$.

The results of this study revealed that the, highest recorded values of marginal gap were in Enamic samples after ageing, $113 \pm 7.01 \mu \mathrm{m}$, with high significant difference between the two subgroups before and after ageing ( $\mathrm{p} \leq 0.001)$, while the two subgroups of Celtra Duo had recorded no significant difference either before or after ageing. The studied reparation designs had recorded no significant difference in subgroups of Celtra Duo. The results are presented numerically and graphically in table 1 and figure 4 .

TABLE (1) Mean and standard deviations of the marginal gap $\mu \mathrm{m}$, before, and after ageing, of the studied preparation designs of both materials of the studied occlusal veneers.

\begin{tabular}{|c|c|c|c|c|}
\hline $\begin{array}{c}\text { Design of } \\
\text { preparation }\end{array}$ & \multicolumn{2}{|c|}{$\begin{array}{c}\text { Celtra groups, } \\
\text { mean } \pm \text { SD }\end{array}$} & \multicolumn{2}{c|}{$\begin{array}{c}\text { Enamic groups, } \\
\text { mean } \pm \text { SD }\end{array}$} \\
\hline $\begin{array}{c}\text { Conventional, } \\
\text { planar }\end{array}$ & Control & Aged & Control & Aged \\
\cline { 2 - 6 } & $80 \pm 10.61$ & $90.45 \pm 13.0$ & $75 \pm 10.43$ & $113 \pm 7.01$ \\
\hline P value & \multicolumn{2}{|c|}{ NS 0.064} & & $0.001 * * *$ \\
\hline $\begin{array}{c}\text { Rounded } \\
\text { shoulder }\end{array}$ & $77.5 \pm 6.61$ & $88.7 \pm 11.60$ & $71 \pm 12.33$ & $105.2 \pm 8.01$ \\
\hline P value & \multicolumn{2}{|c|}{ NS 0.0682} & \multicolumn{3}{|c|}{$0.001 * * *$} \\
\hline
\end{tabular}

$P$ values were significant $* *$ at $\leq 0.05$ 


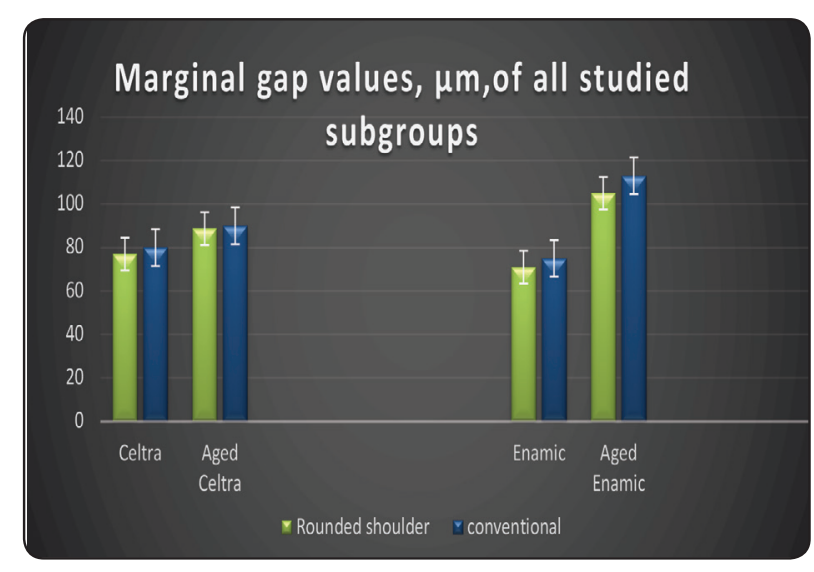

Fig. (3) Histogram showing the values of marginal gap of all studied subgroups, of the occlusal veneers materials.

Based on design and material types, status independent sample t-test was done to compare between marginal gap after ageing in both types of materials, significant considered as $\mathrm{p}<0.05$.

As shown in table (2), although marginal gap values had increased after ageing, yet, no significant difference had recorded between the two preparation designs, in Celtra subgroups, while the results were significant after ageing in Vita Enamic subgroups.

TABLE (2) T-test analysis, showing the effect of ageing and preparation design on the marginal gap, $\mu \mathrm{m}$, of the two studied materials.

\begin{tabular}{|c|c|c|}
\hline & Mean & P value \\
\hline \multirow{3}{*}{ Celtra_after ageing } & $\begin{array}{c}\text { Conventional, planar, } \\
90.45 \pm 13.0\end{array}$ & \multirow{2}{*}{$\leq 0.06$} \\
\cline { 2 - 2 } & $\begin{array}{c}\text { Rounded shoulder } \\
88.7 \pm 11.60\end{array}$ & \\
\hline \multirow{3}{*}{ Enamic_after ageing } & Conventional planar & \\
& $113 \pm 7.01$ & \multirow{2}{*}{$\leq 0.003 * *$} \\
& $\begin{array}{c}\text { Rounded shoulder } \\
105.2 \pm 8.04\end{array}$ & \\
& &
\end{tabular}

Sig. $P \leq 0.05 * *$

The interaction between the studied variables, and their effect on the marginal gap is shown in table (3), it reveals that the highly significant variables were the material type and the ageing process, in addition to preparation together, affecting the marginal gap, while each variable individually, had no influence on violating the marginal adaptation of the studied occlusal veneers.

TABLE (3) Three way Anova test showing the effect of different studied variables, and the interaction between them on vertical marginal gap .

\begin{tabular}{|l|c|c|}
\hline Variables & F ratio & P value \\
\hline Materials & 3.07 & $=0.05$ \\
\hline Design & 0.468 & $0.603 \mathrm{~ns}$ \\
\hline Ageing & 0.732 & $0.753 \mathrm{~ns}$ \\
\hline Material * design & 0.224 & $0.08 \mathrm{~ns}$ \\
\hline Materials *ageing & 12.45 & $0.005^{* *}$ \\
\hline Ageing * design & 0.651 & $0.08 \mathrm{~ns}$ \\
\hline Ageing * materials*design & 10.023 & $0.003 * *$ \\
\hline Adjusted R square $=0.72$ & & \\
\hline
\end{tabular}

\section{Stereo microscope photos:}

Showing representative selective samples of the different, quality, continuity, and the marginal discrepancy, of different occlusal veneers subgroups before and after ageing. Figure 5, A and B representing the occlusal veneers before ageing. C-H representative samples ranging from minute to apparent marginal discrepancy after ageing in different subgroups, of the two materials and preparation designs.

\section{Fourier Transform Infrared Spectroscopy (FT-IR) results}

\section{Celtra Duo spectra :}

The sharp peaks denoting the strong existence of the constituting elements forming the chemical or covalent bonds

As regarding the recorded peaks, numbers and intensity of Celtra Duo samples, a very minute, apparent difference between the two spectra. A and B. 


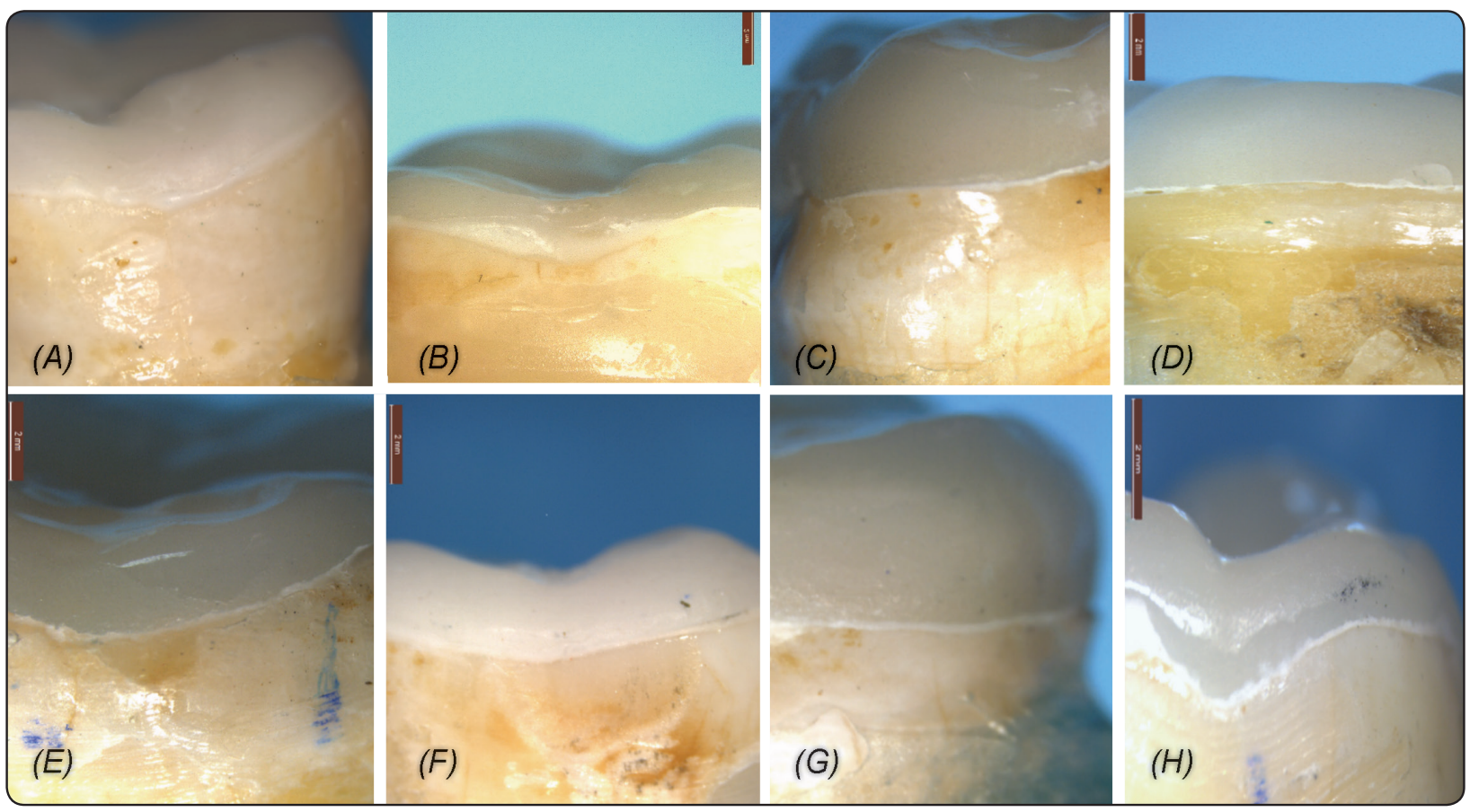

Fig. (5) Representative samples of different marginal quality,control a, and b,after ageing C-H.

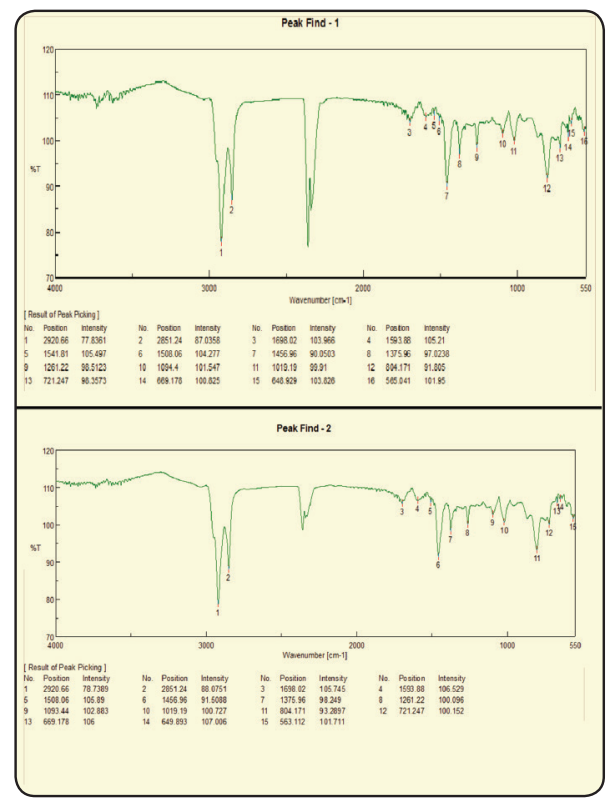

Fig. (6) FT-IR spectra of Celtra occlusal veneer, A, control, B, after ageing

Small and minute decrease in the intensities of different constituting functional bonds, in addition no disappearance, nor blunting or disintegration of any of the peaks in comparison to the control samples. Figure 6.

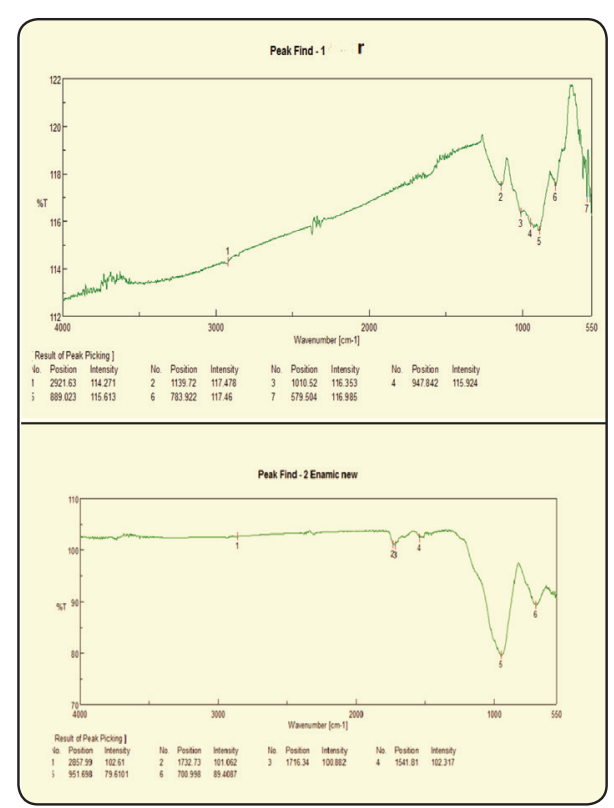

Fig. (7 )FT-IR spectra of Vita Enamic A,control,B,after ageing.

\section{2- Vita Enamic spectra :}

The difference between the two spectra of Vita Enamic is clearly apparent, the stretching vibration of the carbonyl group has a strong infrared absorption between 1710 and $1740 \mathrm{~cm}-1$. Degradation of $\mathrm{C}=\mathrm{O}$ 
and $\mathrm{C}=\mathrm{C}$ bond double bond with, phenyl ring and ester ring, lowers the stretching frequency, which is apparent, in addition there were disappearance of some peaks, denoting breakage of several bonds between the ceramic part and the polymer part of the ceramic. Figure 7.

\section{DISCUSSION}

Achieving esthetically attractive, and functionally ideal restorations, has been the goal of dental clinicians, prosthodontists, and patients, throughout the history of dentistry. Ceramic materials have a natural appearance and excellent biocompatibility, and their clinical use has now been extended to re-habilitation of the complete arch. The success of a dental restoration is determined by many factors among many, the importance of esthetic value, resistance to fracture, and marginal adaptation are mostly addressed as the governing determining factors $^{(16)}$.

Inadequate fit leads to plaque accumulation, which increases the risk of micro leakage, discoloration, and carious lesions. Until the late 1980s, researches who evaluated marginal fit did not always measure the same distances. Holmes et $\boldsymbol{a l}^{(17)}$ proposed a clear terminology in 1989 that include internal gap, marginal gap, overextended margin, under extended margin, vertical marginal discrepancy, horizontal marginal discrepancy, absolute marginal discrepancy and seating discrepancy . Marginal fit is generally evaluated by measuring the marginal gap or the absolute marginal discrepancy ${ }^{(18)}$.

Sever loss of coronal tooth structure or excessive tooth wear is common in the general population (19) .The multifactorial etiology of tooth wear is associated with dietary habits, medical conditions, oral habits and aging that lead to attrition, abrasion, and erosion of the enamel and dentin ${ }^{(20)}$

Treatment of patients with severe erosion or tooth wear is complex. In the past, treatments involved more tooth structure removal which may be inappropriate for patients who have already lost a significant amount of dental tissue due to erosion or attrition. Minimally invasive dentistry has recently become an area of interest. Maximum sound tooth structure conservation is crucial for tooth restorations and, biocompatibility and extended durability. This concept can be applied successfully with occlusal veneers ${ }^{(21)}$, restorations needing less preparation guided by inter occlusal space and teeth anatomy $^{(22)}$.

In this research, the vertical marginal gap of two preparation designs of occlusal veneers constructed of two different ceramic materials had been studied. The first preparation design was the planar, conventional more conservative preparation, guided by the anatomy of the occlusal surface. The depth groove stone was used for standard preparation with $1 \mathrm{~mm}$ of cusp reduction and $1 \mathrm{~mm}$ depth of the central fossa to provide ${ }^{(23,24)}$ enough thickness for the occlusal veneer restoration. The second preparation design had the same occlusal reduction features and, extended $1 \mathrm{~mm}$ on the axial surfaces of the tooth, ended with rounded shoulder finish line. Planar occlusal veneer preparation design have gained a huge reputation over many years as a conservative minimally invasive treatment in severely worn dentition cases, as reported in studies by many authors. Modifications to this design aimed to improve bonding strength of different available materials to tooth structure .The two ceramic materials used to construct the occlusal veneers were ,zirconia reinforced -lithium silicate, ZLS, which comprising a good fatigue resistance comparable to Lithium Disilicate ,in addition to good cementation quality, improved polishing ability, excellent optical properties and translucency ${ }^{(25)}$. The second material, polymer infiltrated ceramics network, PICN. Vita Enamic is a CAD/CAM material that is made of porous presintered feldspar ceramic network matrix (86 wt. $\%)$ infiltrated with organic polymer (14 wt. (\%, in such a combinations, the properties of the material simulates the dentin modulus of elasticity, and with feldspathic ceramic that is similar to enamel. 
ZLS materials have the high Weibull modulus indicates a uniform material quality. Very fine lithium meta silicate and lithium Disilicate crystals with average size $0.5-0.7 \mu \mathrm{m}$. The presence of zirconia provide homogeneous texture to form with a mean grit size of approximately 0.5 to $0.7 \mu \mathrm{m}$. The ceramic have a very fine microstructure that allows a high flexural strength while simultaneously providing a high percentage of glassy matrices. These structural compositions provide the ceramics with good optical and good cementation ${ }^{(26,27)}$.

The occlusal veneers were constructed on a natural molars not on a die materials, in order to be close to the clinical situation. Exposure of restorations, crowns cemented on extracted teeth to cyclic thermal fluctuations to simulate one of the many factors in the oral environment, has been common in many tracer penetration, marginal leakage, marginal gap and bond strength in laboratory tests)..$^{(28)}$ Thermal cycling is one of the most widely used procedures to simulate the physiological aging experienced by biomaterials in clinical practice .Yet, No standards of thermocycling techniques except for, showing only a certain consistency within the temperature parameter $\left(5-55^{\circ} \mathrm{C}\right)$ and a great variability in the number of cycles and dwell time chosen. In this study, thermocycling for 5000 cycles between $5-55^{\circ} \mathrm{C}$ and dwell time 30 seconds ageing was applied on the subgroups of the two materials. Some authors report that in the mouth environment, there are 5000 thermal cycles in five years In addition, immersion of the specimens in food and liquids simulating solutions was also performed ${ }^{(29)}$

A comparisons between the results of vertical marginal gap of the two studied materials revealed that, both materials had initial good marginal fit, although Vita Enamic had a statistically non significant lesser marginal gap $(75 \pm 10.43 \mu \mathrm{m})$, $(71 \pm 12.33 \mu \mathrm{m})$ values, than Celtra Duo specimens $(80 \pm 10.61),(77 \pm 6.61)$, in both, conventional and rounded shoulder preparation designs, respectively. (table 1). Both materials characterized by easy and good milling ability in addition to high edge strength in a relation to the chemical structure of them.

Many investigators reported that rounded shoulder design is preferred than any other finish line configuration ${ }^{(30-32)}$ many studies compared different width of chamfer finish lines with straight shoulder finish lines. Chamfer finish lines yielded better results than straight shoulders. The other articles provided a comparison between shoulder margins with a rounded internal angle and deep, or minimal thickness chamfer finish lines. For various ceramic systems, no significant difference was noted between the 2 finish line designs. On the contrary, studies of others ${ }^{(34-35)}$ showed that minimal thickness finish lines resulted in significantly wider marginal gaps than rounded shoulder as it did not provide the good internal adaptation of the restoration. Non retentive full-coverage occlusal veneers are now a popular treatment option to restore the shape and anatomy of posterior teeth affected by occlusal wear and/or advanced erosive lesions ${ }^{(10)}$ Minimally invasive designs or the "no-preparation" approach with indefinite finish line have many supporters, for teeth where a significant amount of dental tissue has already been lost by wear and erosion, as further tooth preparation may be counterproductive in these cases. However, it may be postulated that shoulder finish line gives more support than indefinite finish line as in the conventional design, Moreover, the cutting tools of the milling machine in CAD CAM system has a diameter that requires a minimum of $0.3 \mathrm{~mm}$ of the circumferential edge of the restoration, a condition which may not be possible in the conventional planar design. So this may give rise to the slightly increased values of the marginal gap in the conventional design. After ageing, the results had shown a high discrepancy in marginal adaptation of Vita Enamic subgroups, as marginal gap values recorded were $113 \pm 7.01$ and $105.2 \pm 8.01 \mu \mathrm{m}$ at the conventional and rounded shoulder designs respectively, $\mathrm{p} \leq 0.003$. While in Celtra Duo subgroups, the violation in marginal adaptation was less than in Enamic specimens 
recording $90.45 \pm 13.0$ and $88.7 \pm 11.60 \mu \mathrm{m}$ at the conventional and rounded shoulder designs respectively, with no significant difference between the two preparation designs. It seems that the ceramic materials has seldom negative affection by the ageing protocol of this study. These results were in agreement with Daniele A.et al ${ }^{(36)}$ how studied the influence of preparation designs on marginal adaptation and failure load of full-coverage occlusal veneers after thermo-mechanical aging simulation . They evaluated the fracture resistance and marginal quality of maxillary molars restored using lithium disilicate glass-ceramic (LDG) occlusal veneers with two finish line thickness; minimal chamfer and rounded shoulder. After ageing they measured the fracture load and the marginal discrepancy. The results showed that the thermocycling ageing did not had a significant effect on the lithium disilicate glass, fracture load, or its good marginal seal. The internal structure of LDG nearly approximate the chemical structure of ZLS occlusal veneers used in the current study. On the contrary, Vita Enamic which is polymer infiltrated ceramic network, PICN, had apparent violation in marginal adaptation. The FT-IR spectra of the material showing that many peaks had degraded, the intensities of the $\mathrm{C}=\mathrm{OH}$ and $\mathrm{C}=\mathrm{C}$ had decreased and degraded.

The temperature gradient is created as a result of heating and cooling of the surface element, on the other hand, the surface and core of the material remaining in the force interaction, trying to counter act and inhibit the change of surface dimensions ${ }^{(37)}$.

Thermal fatigue mainly, results from mechanical deformation after subjected to cycles of heating and cooling, in the material by the two acting bonds. These bonds can be classified as internal or external ${ }^{(38)}$. External bonds include all elements that restrict the deformation of the material such as strong cementation bond between the tooth substrate and the restoration. Internal bonds can result from anisotropic structure, temperature gradient, and phases with different coefficients of thermal expansion. In polymer-ceramic composites, the filler and the matrix have different coefficients of thermal expansion. It could be seen that hydrolytic aging of polymer-ceramic composites occurs, but destruction mechanisms have not yet been clearly identified. Many reported researches pointed out that water in a polymer matrix composite structure acts like a plasticizer, so deformation of the materials takes place ${ }^{(38-41)}$. It is also probable that chemical compounds decompose the polymer network in the aqueous solution by disintegrating ester bonds ${ }^{(37)}$.

In addition, immersion in food and liquid simulating solutions seems to have also a great effect on the Vita Enamic integrity. It is well known that citric acid affects both the polymer part and the filler part as well. When the PICN specimens exposed to low $\mathrm{pH}$ environment, the inorganic fillers tend to fall out from resin material and the matrix components decompose. Consequently, leakage of filler constituents has been shown to produce cracks at the resin- filler interface, which may lead to weakening of the material ${ }^{(42)}$. In addition, The $75 \%$ ethanol solution has high solubility effect to BisGMA-based materials, and the closer the solubility effect between solvent and solute, the greater the solute's degradation ${ }^{(42)}$. Compositional analysis of PICN showed a dominant ceramic-network with leucite as the major phase and zirconia as a minor phase interconnected with a polymer-based network mainly Bis-GMA. Thermocycling is a combination of hydrolytic and thermal degradation and a method to simulate temperature-related breakdown by repeated sudden temperature changes ${ }^{(43)}$. Prolonged thermocycling may lead to water absorption by a diffusion-controlled process, and it causes leaching of unreacted monomers and swelling of the matrix.

The results of the current study showed that after ageing the design (2) with rounded shoulder and $1 \mathrm{~mm}$ extension on the axial walls recorded better adaptation with less marginal gap. (Table 1).Although the results not significant between the two designs, yet, it may be a clue that this design 
offered more surface area for bonding in addition to the good internal support of the rounded shoulder finish line. The values of marginal gap of the two studied materials even after ageing were within the clinical accepted marginal gap, $(90.45 \pm 13.0$, and $88.7 \pm 11.60 \mu \mathrm{m}$ ) for Celtra subgroups at conventional, and rounded shoulder respectively, while Vita Enamic subgroups recorded $(113 \pm 10.01$, and $105.2 \pm 11.04 \mu \mathrm{m}$ ) at the conventional and rounded shoulder designs respectively. The American Dental Association states in Specification No. 8 that the film thickness of the zinc phosphate cement should not exceed $25 \mu \mathrm{m}-40 \mu \mathrm{m}$ for different cements types ${ }^{(44)}$

However, several studies that examined marginal adaptation have shown that this goal was seldom reached $25 \mu \mathrm{m}$. Gardne ${ }^{(45)}$ observed that, even under ideal conditions, it was very rare to achieve this level of accuracy. After a 5-year study conducted on more than 1000 restorations, McLean and Von Fraunhaufer $^{(46)}$ concluded that $120 \mu \mathrm{m}$ was the maximum tolerable marginal. The marginal gap values of the current study were considered accepted clinically according the reported values of many studies and review of the data retrieved for marginal gap that showed nearly $94.9 \%$ of the values measured in the literatures were less than or equal to $120 \mathrm{~mm}$. While others reported much more values than these. The widest marginal gap measured was $174 \mathrm{~mm}$, and the smallest was $3.7 \mathrm{~mm}^{(36,47-50)}$.

The inter action between the material types and the ageing and the preparation design had shown a high significance on influencing the marginal gap values (table3), thus, the clinicians must be careful when choosing specific material for specific clinical situation that requires certain preparation designs, as the longevity of good marginal seal may be, questionable.

From the previous results and discussion, we may say that the null hypothesis of the current study was rejected.
Further studies should be performed on these conservative restorations, regarding the longevity and fracture strength of different materials and other preparation designs.

\section{CONCLUSIONS}

1- The choice of certain preparation design should be associated with meticulous knowledge of the material of the occlusal veneers properties.

2- Both materials could be used in occlusal veneers restorations.

3- Celtra Duo had a marginal adaptation that survived with better qualitatively and quantitatively values, even after the protocol of ageing applied in this study.

\section{REFERENCES}

1. Abrahamsen TC. The worn dentition-pathognomonic patterns of abrasion anderosion. Int Dent J 2005; 55:268-76.

2. Turner KA, Missirlian DM. Restoration of the extremely worn dentition. J Prosthet Dent 1984; 52:467-74.

3. Al-Omiri MK, Lamey PJ, Clifford T. Impact of tooth wear on daily living. Int J Prosthodont 2006; 19:601-5.

4. Moslehifard E, Nikzad S, Geraminpanah F, et al. Fullmouth rehabilitation of a patient with severely worn dentition and uneven occlusal plane: a clinical report. J Prosthodont 2012; 21:56-64.

5. Edelhoff D, Sorensen JA. Tooth structure removal associated with various preparation designs for posterior teeth. Int J Periodontics Restorative Dent 2002; 22:241-9.

6. Edelhoff D, Sorensen JA. Tooth structure removal associated with various preparation designs for anterior teeth. J Prosthet Dent 2002; 87:503-9.

7. Magne P, Belser U. Bonded porcelain restorations in the anterior dentition: a biomimetic approach. Chicago: Quintessence; 2002. p. 23-55.

8. Torbjorner A, Fransson B. Biomechanical aspects of prosthetic treatment of structurally compromised teeth. Int $\mathbf{J}$ Prosthodont 2004; 17:135-41.

9. Magne P, Schlichting LH, Maia HP, Baratieri LN. In vitro fatigue resistance of CAD/CAM composite resin and ceramic posterior occlusal veneers. J Prosthet Dent 2010; 104:149-57. 
10. Schlichting LH, Maia HP, Baratieri LN, Magne P. Noveldesign ultra-thin $\mathrm{CAD} / \mathrm{CAM}$ composite resin and ceramic occlusal veneers for the treatment of severe dental erosion. J Prosthet Dent 2011:217-26.

11. Oh, S.C., Dong, J.K., Luthy, H., Scharer, P. Strength and microstructure of IPS Empress 2 glass-ceramic after different treatments. Int. J. Prosthodont. 2000; 13, 468-472.

12. Elsaka, Shaymaa E., and Amr M. Elnaghy. Mechanical properties of zirconia reinforced lithium silicate glassceramic. Dent. Mater.2016; 32: 908-14.

13. Coldea, A., Swain, M.V., Thiel, N. Mechanical properties of polymer-infiltrated ceramic-network materials. Dent. Mater. 2013; 29, 419-26.

14. Vouvoudi EC, Sideridou ID. Effect of food/oral-simulating liquids on dynamic mechanical thermal properties of dental nano hybrid light-cured resin composites. Dent Mater.; 2013 Aug; 29(8):842-50.

15. Yesilyurt C, Yoldas O, Altintas SH, Kusgoz A. Effects of food-simulating liquids on the mechanical properties of a silorane- based dental composite. Dent. Mater. J. 2009; 28(3):362-7.

16. Mathieu C., Arnaud S., Michel B., and Odile L.: Marginal adaptation of ceramic crowns :A systematic review. J Prosthet Dent 2013;110:447-454

17. Holmes JR, Bayne SC, Holland GA,Sulik WD. Considerations in measurement of marginal fit. J Prosthet Dent 1989;62:405-8.

18. Martinez-Rus F, Ferreiroa A, Ozcan M, Pradies G. Marginal discrepancy of monolithic and veneered all-ceramic crowns on titanium and zirconia implant abutments before and after adhesive cementation: a scanning electron microscopy analysis. Int J Oral Maxillofac Implants 2013;28:480-7.

19. Van't Spijker A, Rodriguez JM, Kreulen CM, et al. Prevalence of tooth wear in adults. Int $\mathrm{J}$ Prosthodont 2009;22:35-42.

20. Edelhoff D, Sorensen JA: Tooth structure removal associated with various preparation designs for anterior teeth. $\mathrm{J}$ Prosthet Dent. 2002; 87(5): 503-509.

21. Pelekanos S, Koumanou M, Koutayas SO, Zinelis S, Eliades G. Micro-CT evaluation of the marginal fit of different In-Ceram alumina copings. Eur J Esthet Dent 2009; 4:278-92.

22. Jonathon S. Egberta, Andrew C. Johnsonb, Daranee Tantbirojnc, Antheunis Versluisd : Fracture strength of ultrathin occlusal veneer restorations made from CAD/CAM composite or hybrid ceramic materials . Oral Science International 2015;12:53-58.
23. Magne P, Schlichting LH, Maia HP, Baratieri LN. In vitro fatigue resistance of $\mathrm{CAD} / \mathrm{CAM}$ composite resin and ceramic posterior occlusal veneers. J Prosthet Dent. 2010;104:149-157.

24. Sorrentino R, Nagasawa Y, Infelise M, et al. In vitro analysis of the fracture resistance of CAD-CAM monolithic lithium disilicate molar crowns with different occlusal thickness. J Osseointegr. 2018;10: 24

25. Sven R, Matthias Rö., Dirk Zi. , and Anne-Kathrin Sc.: Fabrication of Zirconia-Reinforced Lithium Silicate Ceramic Restorations Using a Complete Digital Workflow; Case Reports in Dentistry Volume 2015, 2015: 162178

26. Denry and J. R. Kelly, "Emerging ceramic-based materials for dentistry," Journal of Dental Research, vol. 93, no. 12, pp. 1235-1242, 2014.

27. Andrade JP, Stona D, Bittencourt HR, Borges GA, Burnett LH Júnior, Spohr AM. Effect of different computer-aided design/computer-aided manufacturing (CAD/CAM) materials and thicknesses on the fracture resistance of occlusal veneers. Oper Dent. 2018;43:539-548.

28. Anna LuciaMorresia, MaurizioD'Amarioa, n, Mario Capogrecoa, Roberto Gattob, GiuseppeMarzob, CamilloD’ Arcangeloc AnnalisaMonaco. Thermal cycling for restorative materials: Does a standardized protocol exist in laboratory testing? A literature review Journal of the mechanical behavior of biomedical materials 29(2014)295-308.

29. Ahmed M. Sayed, Maha A. Niazy and Inas T. Motawee. Effect of Food Simulating Liquids and Thermo-cycling on flexural strength and surface hardness of a Resin NanoCeramic CAD/CAM Material ; ADJ-for Girls, Vol. 3, No. 2, April (2016) - PP. 125:133

30. Castillo Oyague R, Sanchez-Jorge MI,Sanchez Turrion A. Influence of CAD/CAM scanning method and toothpreparation design on the vertical misfit of zirconia crown copings. Am J Dent 2010;23:341-6.

31. Ural C, Burgaz Y, Sarac D. In vitro evaluation of marginal adaptation in five ceramic restoration fabricating techniques. Quintessence Int 2010;41:585-90.

32. Tao J, Han D. The effect of finish line curvature on marginal fit of all-ceramic CAD/CAM crowns and metal-ceramic crowns. Quintessence Int 2009;40:745-52.

33. Fahmy NZ. Influence of veneering materials on the marginal fit and fracture resistance of an alumina core system. J Prosthodont 2011;20:45-51.

34. D'Arcy BL, Omer OE, Byrne DA, Quinn F.The reproducibility and accuracy of internal fit of Cerec 3D CAD/ CAM all ceramic crowns. Eur J Prosthodont Restor Dent 2009;17:73-76 
35. Comlekoglu M, Dundar M, Ozcan M, Gungor M, Gokce B, Artunc C. Influence of cervical finish line type on the marginal adaptation of zirconia ceramic crowns. Oper Dent 2009;34:586-92.

36. Daniele Angerame ,Matteo De Biasi I,Martina Agostinetto I Andrea Franzò , and Giulio Marchesi Influence of preparation designs on marginal adaptation and failure load of full-coverage occlusal veneers after thermomechanical aging simulation; J Esthet Restor Dent. 2019;1-10

37. Daniel Pieniak, Krzysztof Przystupa, AgataWalczak, Agata M. Niewczas, Aneta Krzyzak , Grzegorz Bartnik , Leszek Gil and Paweł Lonkwic ; Hydro-Thermal Fatigue of Polymer Matrix Composite Biomaterials. Materials 2019, 12, 3650; doi:10.3390/ma12223650.

38. Al-wahab ZN. An evaluation of the effect of different solutions on the micro hardness of aesthetic restoration. MDJ. 2011;8(2):106-14.

39. Paula AB De, Caroline R, Alonso B, Albamonte G, Araújo $\mathrm{S}$ De, Rontani JP, et al. Influence of chemical degradation and abrasion on surface properties of nano restorative materials. Braz Dent Sci. 2015;14(2):100-5.

40. Vouvoudi EC, Sideridou ID. Effect of food/oral-simulating liquids on dynamic mechanical thermal properties of dental nanohybrid light-cured resin composites. Dent Mater. The Academy of Dental Materials; 2013 Aug; 29(8):842-50.

41. Akova T, Ozkomur A, Uysal H. Effect of food-simulating liquids on the mechanical properties of provisional restorative materials. Dent Mater2006;22:1130-4.

42. Peampring C, Sanohkan S. Effect of Thermocycling on Flexural Strength and Weibull Statistics of Machinable
Glass-Ceramic and Composite Resin. J Indian Prosthodont Soc . 2013;14(4):1-5.

43. Julia Cadorim Fa. ,Marcia B., Pedro Henrique Corazza. A literature review on the new polymer-infiltrated ceramicnetwork material (PICN). J Esthet Restor Dent. 2018;1-6.

44. American Dental Association. ANSI/ADA Specification no.8 for zinc phosphate cement. In: Guide to dental materials and devices. $5^{\text {th }}$ ed. Chicago: American Dental Association; 1970-71.

45. Gardner FM. Margins of complete crowns literature review. J Prosthet Dent1982;48:396-400.

46. McLean JW, von Fraunhofer JA. The estimation of cement film thickness by an in vivo technique. Br Dent $\mathrm{J}$ 1971;131:107-11.

47. Bhowmik H, Parkhedkar R. A comparison of marginal fit of glass infiltrated alumina copings fabricated using two different techniques and the effect of firing cycles over them. J Adv Prosthodont 2011;3:196-203.

48. Lee KB, Park CW, Kim KH, Kwon TY. Marginal and internal fit of all-ceramic crowns fabricated with two different CAD/CAM systems. Dent Mater J 2008;27:422-6.

49. Iwai T, Komine F, Kobayashi K, Saito A, Matsumura $\mathrm{H}$. Influence of convergence angle and cement space on adaptation of zirconium dioxide ceramic copings. Acta Odontol Scand 2008;66:214-8

50. Baroudi K, Ibraheem SN. Assessment of chair-side computer-aided design and computer-aided manufacturing restorations: a review of the literature. J Int Oral Health 2015;7:96-104. 